\title{
Outstanding passivation effect by a new mixed-salt interlayer in perovskite solar cells
}

Bowen Yang, ${ }^{\mathrm{a} \dagger}$ Jiajia Suo, ${ }^{\mathrm{a} \dagger}$ Edoardo Mosconi, ${ }^{\mathrm{b}}$ Damiano Ricciarelli, ${ }^{\mathrm{b}, \mathrm{c}}$ Wolfgang Tress, ${ }^{\mathrm{d}}$ Filippo De Angelis, ${ }^{\text {b,ce }}$ Hui-Seon Kim, ${ }^{\mathrm{f}^{*}}$ and Anders Hagfeld $\mathrm{t}^{\mathrm{a}}$

aLaboratory of Photomolecular Science, Institute of Chemical Sciences and Engineering, School of Basic Sciences, Ecole Polytechnique Fédérale de Lausanne, CH-1015 Lausanne, Switzerland.

${ }^{b}$ Computational Laboratory for Hybrid/Organic Photovoltaics (CLHYO), Istituto CNR di Scienze e Tecnologie Chimiche "Giulio Natta" (CNR-SCITEC), Via Elce di Sotto 8, 06123 Perugia, Italy.CNR-ISTM, 06123 Perugia, Italy.

${ }^{c}$ Department of Chemistry, Biology and Biotechnology, University of Perugia, Via Elce di Sotto 8, 06123 Perugia, Italy.

${ }^{\mathrm{d}}$ Institute of Computational Physics, Zurich University of Applied Sciences, Wildbachstr. 21, 8401 Winterthur, Switzerland.

${ }^{\text {e}}$ CompuNet, Istituto Italiano di Tecnologia, Via Morego 30, 16163 Genova, Italy.

fDepartment of Chemistry, Inha University, Incheon 22212, Korea.

$\dagger$ Both authors contributed equally to this work.

* Corresponding authors

H.-S.K.: hui-seon.kim@inha.ac.kr and A. H.: anders.hagfeldt@epfl.ch 


\begin{abstract}
Non-radiative recombination is a major limit in obtaining efficient metal-halide perovskite photovoltaics. Here, a passivation treatment is developed by applying a mixture of a new ammonium salt, $\mathrm{CF}_{3}\left(\mathrm{CF}_{2}\right)_{2} \mathrm{CH}_{2} \mathrm{NH}_{3} \mathrm{I}$ (HBAI), and a conventional formamidinium halide, $\mathrm{FAX}$, on top of the 3D perovskite bulk film. The introduction of such an optimized passivation layer effectively suppressed charge recombination by passivating the surface traps of the 3D perovskite film, enabling a well-matched energy alignment at the interface of perovskite/HTM, leading to a high power conversion efficiency (PCE) of over 23\% from current (I)-voltage (V) measurements and a stabilized $22.7 \%$ PCE, owing to a substantial $V_{\mathrm{OC}}$ improvement (above 80 $\mathrm{mV}$ in average), coupled with an improved stability.
\end{abstract}



Metal halide perovskites have attracted significant interests due to their outstanding optoelectronic properties and obtained successive improvements especially in photovoltaics, leading to high power conversion efficiency (PCE) of the perovskite solar cells (PSCs). ${ }^{1,2}$ The first PSC contained a liquid electrolyte, which attained 3.8\% of power conversion efficiency (PCE). ${ }^{3}$ Nevertheless, immediate attention to PSC was not raised owing to its instability, where the liquid electrolyte readily dissolves the halide perovskite. ${ }^{4}$ Subsequently, the development of solid-type PSCs based on a small-molecule hole transport material (HTM) made an important breakthrough in 2012,,$^{1,2}$ showing around 10\% of PCE with 500 hour-long shelf stability in ambient air. ${ }^{1}$ Since then, extensive efforts have been placed on the PSCs and thus achieved significant improvements from the perspective of both performance and fundamental understandings. ${ }^{5-8}$ A state-of-the-art technology of PSCs can afford to reach a remarkable record efficiency of $25.2 \%$ in $2020 .{ }^{9}$ However, this is still far behind the theoretical limit of $32 \%$, defined by the Shockley-Queisser theory with $1.50 \mathrm{eV}$ of bandgap, which is mainly ascribed to the substantial non-radiative recombination loss in PSCs. ${ }^{10-12}$ In order to hinder the non-radiative recombination in devices, several approaches have been employed such as defect engineering and interface passivation. ${ }^{11-16}$ On one hand, morphological and compositional engineering have been extensively explored to gain an ideally high-quality of defect-less perovskite film. ${ }^{6,17}$ On the other hand, foreign molecules are employed either in the perovskite layer or on top of the perovskite layer, ${ }^{18-21}$ aiming at passivating defects in the perovskite bulk layer or on its surface. It is found that a moderate excess of $\mathrm{PbI}_{2}$ in the perovskite film not only results in favorable bigger perovskite crystallites with higher crystallinity, but also reduces the defect-assisted recombination at the perovskite $/ \mathrm{TiO}_{2}$ interface. ${ }^{22-25} \mathrm{In}$ addition, a variety of organic molecules have been tried as passivation materials in PSCs. ${ }^{26-31} \mathrm{~A}$ remarkable PCE of $23.32 \%$ was achieved by adopting phenethylammonium iodide (PEAI) as a passivation layer. Its impressive $V_{\mathrm{OC}}$ of $1.18 \mathrm{eV}$ corresponds to $94 \%$ of the theoretical $V_{\mathrm{OC}}$ estimated by the Shockley-Queisser (SQ) limit ${ }^{26}$ which indicates the maximum theoretical efficiency obtained from a single $p-n$ junction under the ideal case without non-radiative recombination. Similarly, an ultrahydrophobic 2D passivation layer was obtained from pentafluorophenylethylammonium lead iodide, $(\mathrm{FEA})_{2} \mathrm{PbI}_{4}$, on top of the $3 \mathrm{D}$ perovskite, demonstrating a PCE over $22 \%$ with $V_{\mathrm{OC}}$ of $1.10 \mathrm{~V}^{27}$

Herein, we develop a highly efficient PSC by designing a new mixed-salt interlayer for the purpose of passivating the $3 \mathrm{D}$ perovskite film. While the previous works mostly focused on the passivation effect through organic molecule or 2D perovskite capping layer, this study aims at 
achieving not only passivation effect but also efficient energy level alignment by using the benefits of two different salts with internal interaction. The internal interaction between the mixed salts plays an important role which is worth being considered when devising the molecules for passivation. Accordingly, a thin passivation layer is formed by a mixed salts of a novel ammonium salt, $\mathrm{CF}_{3}\left(\mathrm{CF}_{2}\right)_{2} \mathrm{CH}_{2} \mathrm{NH}_{3} \mathrm{I}$ (HBAI) and the conventional formamidinium halide (FAX, where $\mathrm{X}$ is $\mathrm{I}, \mathrm{Br}$, or $\mathrm{Cl}$ ). The introduction of the optimized passivation layer effectively reduces the surface defects of the 3D perovskite film with efficient charge extraction, showing a PCE of $23.1 \%$ from the current (I)-voltage (V) curve and a stabilized $22.7 \%$ from the maximum power point (mpp) due to a significant $V_{\mathrm{OC}}$ increase, coupled with an improved stability.

A perovskite film composed of $\mathrm{Cs}_{0.05} \mathrm{MA}_{0.1} \mathrm{FA}_{0.85} \mathrm{PbI}_{2.9} \mathrm{Br}_{0.1} \cdot 0.05 \mathrm{PbI}_{2}$, was chosen for the ensured high quality of the film. ${ }^{30}$ The perovskite bulk film (photoactive 3D) was prepared by a one-step spin-coating method where chlorobenzene was dripped as an anti-solvent at the end of spinning, followed by 1 hour annealing at $100{ }^{\circ} \mathrm{C}$. An additional passivation layer was deposited on the as-prepared 3D perovskite film after being cooled down to room temperature by spin-coating a solution containing mixed-salts, a novel fluorine-rich ammonium salt of HBAI and FAX (X = I, Br or Cl), which was sequentially post-annealed. A schematic device architecture and the molecular structures of HBAI and FAX are shown in Figure 1.

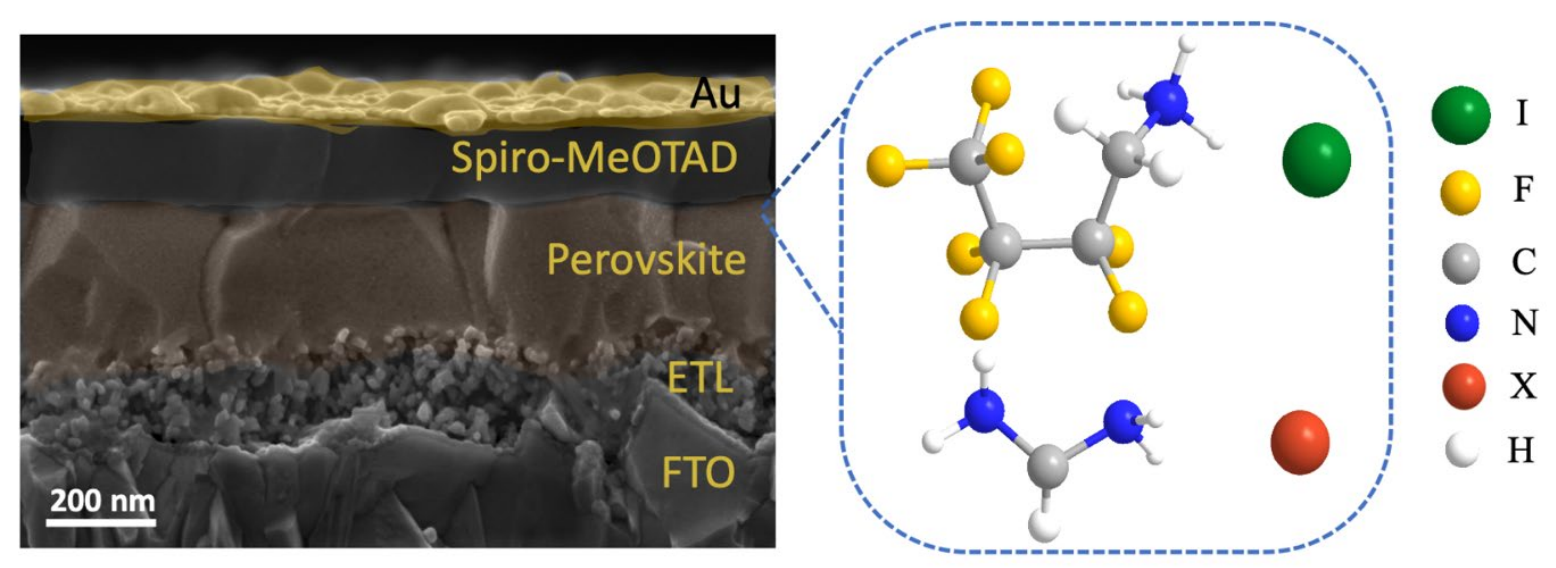

Figure 1 Schematic illustration of the device structure (left) and molecular structures of HBAI and FAX (right) used for the passivation layer, where $\mathrm{X}=\mathrm{I}, \mathrm{Br}$, or $\mathrm{Cl}$.

As shown in Figure 1, the mixed passivation layer was employed in the n-i-p architecture of $\mathrm{FTO} / c p-\mathrm{TiO}_{2} / m p-\mathrm{TiO}_{2} / 3 \mathrm{D}$ perovskite/passivation layer/spiro-MeOTAD/Au. Remarkably, 
more superior photovoltaic performance is observed when HBAI is accompanied with FAX for the passivation layer, compared to the exclusive use of HBAI (see Figure S1). Statistical box charts of the photovoltaic parameters are shown in Figure 2, where the molar ratio between HBAI and FAX was fixed to 1:1. The corresponding average values of Figure 2 are summarized in Table 1. Compared to control devices, the devices employing HBAI·FAX passivation treatment resulted in better performance throughout all the compositions regardless of the halide in FAX, particularly in open circuit voltage $\left(V_{\mathrm{OC}}\right)$ and fill factor $(\mathrm{FF})$, leading to a significant improvement in PCE. The photocurrent density $\left(J_{\mathrm{SC}}\right)$ shows comparable value around $25.2 \mathrm{~mA} / \mathrm{cm}^{2}$ and corresponding incident photon-to-current efficiency (IPCE) are shown in Figure S2, where the tendency of integrated $J_{\mathrm{SC}}$ is well matched with $J_{\mathrm{SC}}$ from I-V curves. However, $V_{\mathrm{OC}}$ of $1.06 \mathrm{~V}$ from the control device is greatly increased to $1.14 \mathrm{~V}$ for HBAI·FAI, $1.15 \mathrm{~V}$ for HBAI·FABr and $1.12 \mathrm{~V}$ for HBAI·FACl. Furthermore, FF is also notably enhanced by mixed-HBAI·FAX treatment, showing 0.78 for both FAI and FACl and 0.79 for FABr. Accordingly, the mixed-salt of HBAI-FABr leads to the highest average PCE of $22.78 \%$, while the control device demonstrates the average PCE of $20.10 \%$.
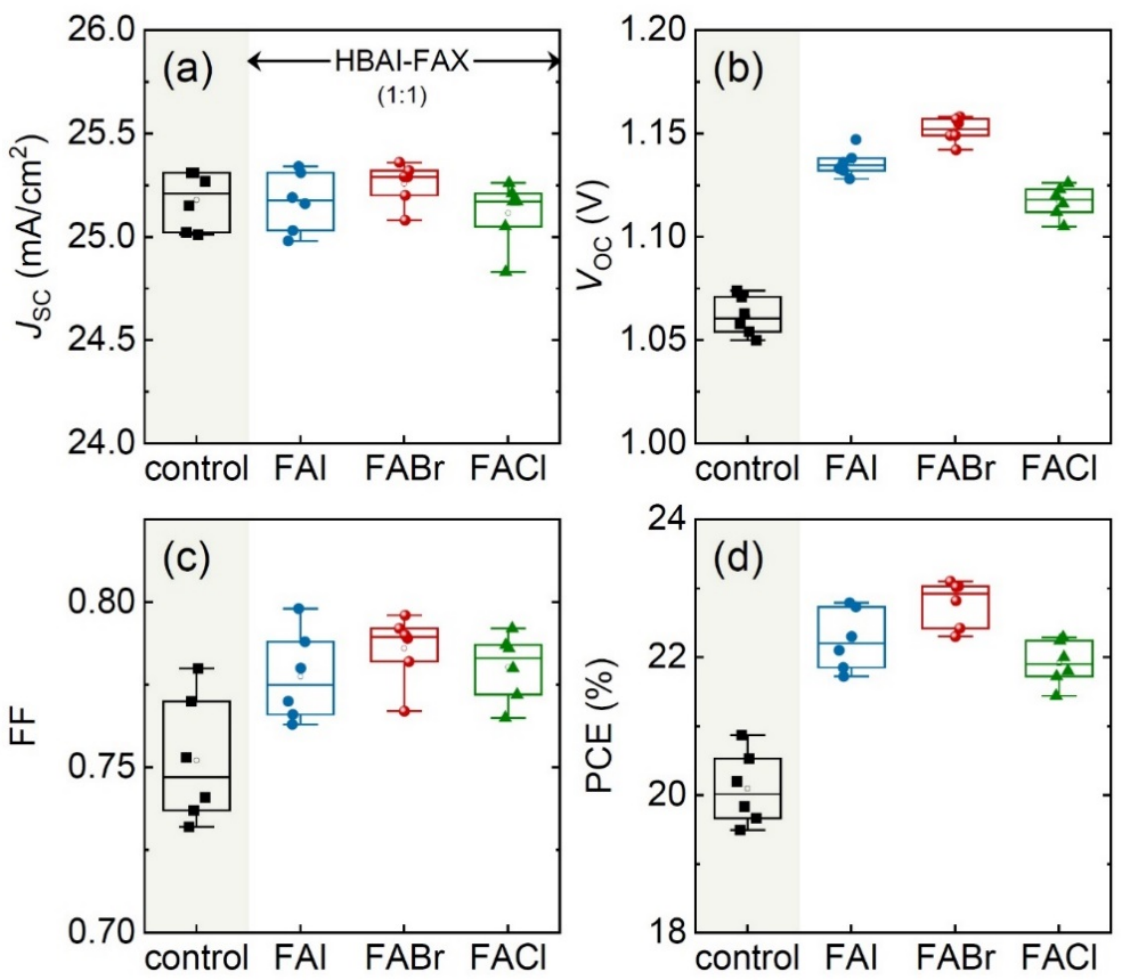

Figure 2 Statistical box charts for the photovoltaic parameters of (a) $J_{\mathrm{SC}}$, (b) $V_{\mathrm{OC}}$, (c) FF and 
(d) PCE of devices without a passivation layer (control, black) and with a passivation layer of mixed HBAI·FAX. HBAI·FAI (blue), HBAI·FABr (red), and HBAI·FACl (green). The devices were measured under AM $1.5 \mathrm{G}$ solar irradiance at $100 \mathrm{~mW} / \mathrm{cm}^{2}$ with a voltage sweep of the reverse scan (RS, from open circuit to short circuit condition).

Table 1 Average photovoltaic parameters of PSCs with and without passivation layers based on different salt compositions, HBAI·FAX.

\begin{tabular}{ccccc}
\hline $\begin{array}{c}\text { Salt composition for } \\
\text { the passivation layer }\end{array}$ & $\begin{array}{c}J_{\mathrm{SC}} \\
\left(\mathrm{mA} / \mathrm{cm}^{2}\right)\end{array}$ & $\begin{array}{c}V_{\mathrm{OC}} \\
(\mathrm{V})\end{array}$ & $F F$ & $\begin{array}{c}P C E \\
(\%)\end{array}$ \\
\hline control & 25.18 & 1.06 & 0.75 & 20.10 \\
\hline $\mathrm{HBAI} \cdot \mathrm{FAI}$ & 25.17 & 1.14 & 0.78 & 22.25 \\
\hline $\mathrm{HBAI} \cdot \mathrm{FABr}$ & 25.26 & 1.15 & 0.79 & 22.78 \\
\hline $\mathrm{HBAI} \cdot \mathrm{FACl}$ & 25.12 & 1.12 & 0.78 & 21.92 \\
\hline
\end{tabular}

The effect of the HBAIX $\cdot \mathrm{FABr}_{1-\mathrm{X}}$ mixed-salt on the photovoltaic parameters (Figure S1) was examined with varying the HBAI ratio $(X=0,0.25,0.50,0.75$ and 1.00$)$, however maintaining the same total molarity. Notably, the device performances are both improved while applying only FAX or HBAI, however, more superior performance is observed with the mixture. While $J_{\mathrm{SC}}$ shows a negligible difference depending on the composition of $\mathrm{HBAI}_{\mathrm{X}} \cdot \mathrm{FABr}_{1-\mathrm{X}}$ (Figure S1(a)), an obvious change is monitored from $V_{\mathrm{OC}}$ and FF. The highest average $V_{\mathrm{OC}}$ is observed from $\mathrm{HBAI}_{0} \cdot \mathrm{FABr}_{1}$ and gradually decreases with increasing $\mathrm{HBAI}$ ratio in $\mathrm{HBAI}_{\mathrm{X}} \cdot \mathrm{FABr}_{1-\mathrm{X}}$, where the $V_{\mathrm{OC}}$ from $\mathrm{HBAI}_{1} \cdot \mathrm{FABr}_{0}$ is still higher than that of control devices without the passivation layer (Figure S1(b)). On the other hand, FF is mainly governed by HBAI, showing the highest FF by the equivalent contribution of HBAI and $\mathrm{FABr}(\mathrm{X}=0.5)$ to the mixed salts (Figure S1(c)). Therefore, the highest average $\mathrm{PCE}$ is obtained from $\mathrm{HBAI}_{0.5} \cdot \mathrm{FABr}_{0.5}$ (Figure S1(d)), which confirms the optimized ratio of 1:1 in Figure 2. In Figure 3(a) the current (I)voltage (V) curves of the control device and the HBAI FABr-based device are compared. It is notable that the HBAI-FABr-based device results in a low hysteresis index (HI) of $1.5 \%$, defined as $\left(\mathrm{PCE}_{\text {reverse- }} \mathrm{PCE} \mathrm{E}_{\text {forward }}\right) / \mathrm{PCE}_{\text {reverse, }}{ }^{32}$ while the control device suffers from a severe hysteresis. A prominent improvement in $V_{\mathrm{OC}}$ and $\mathrm{FF}$ is found from the HBAI-FABr-based device, leading to $23.10 \%$ of PCE based on $25.11 \mathrm{~mA} / \mathrm{cm}^{2}$ of $J_{\mathrm{SC}}, 1.16 \mathrm{~V}$ of $V_{\mathrm{OC}}$ and 0.79 of FF from the reverse scan (RS). The stabilized PCE at maximum power point was indeed 
measured and reached $22.7 \%$ as shown in Figure 3(b). Furthermore, IPCE spectra in Figure 3(c) ensures the efficient light harvesting and charge collection throughout the broad absorbance window of the 3D perovskite. The integrated IPCE is in accordance with the $J_{\mathrm{SC}}$ value from the I-V curve.
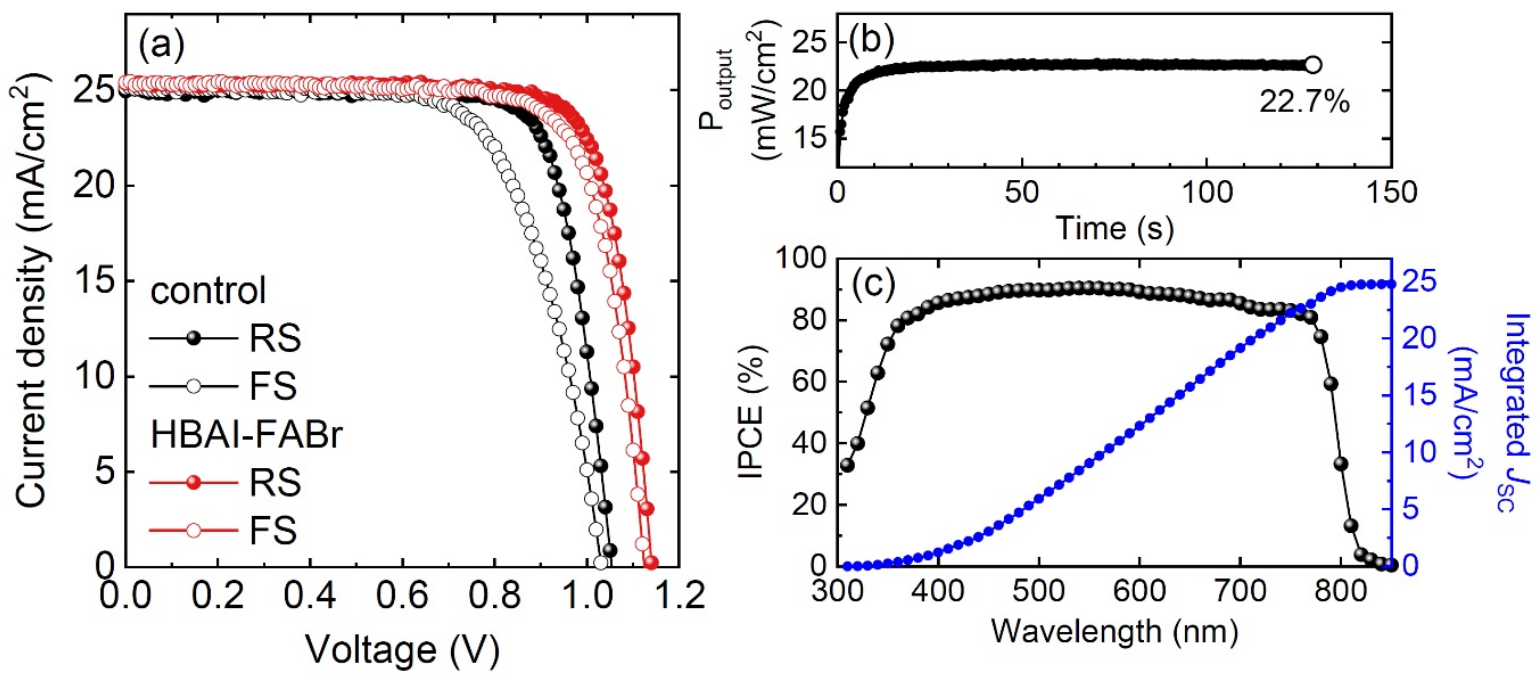

Figure 3 (a) I-V curves of the control (black) and HBAI·FABr (red) devices. Reverse scan (RS) and forward scan (FS) are indicated as solid symbols and open symbols, respectively. (b) Power output of the device employing a $\mathrm{HBAI} \cdot \mathrm{FABr}$ passivation layer at maximum power point as a function of time. (c) IPCE and integrated photocurrent density of the device employing a HBAI $\cdot$ FABr passivation layer.

High resolution scanning electron microscope (HR-SEM) was performed to understand the effect of the mixed-salt (HBAI·FABr) on the morphology of the perovskite layer. The surface images of the control perovskite film and the post-treated film with HBAI·FABr are shown in Figures 4(a) and 4(b), respectively. In the control sample, a dense polycrystalline perovskite film is formed with white crystals, indicative of excessive $\mathrm{PbI}_{2}$ presents on the surface and in the grain boundaries. ${ }^{25}$ When the pristine perovskite is treated with HBAI FABr, the exposed $\mathrm{PbI}_{2}$ on the surface is no longer observed, resulting in an enhanced film quality in homogeneity and uniformity. The removal of the $\mathrm{PbI}_{2}$ would reduce the series resistance which explains the increase of the FF. 

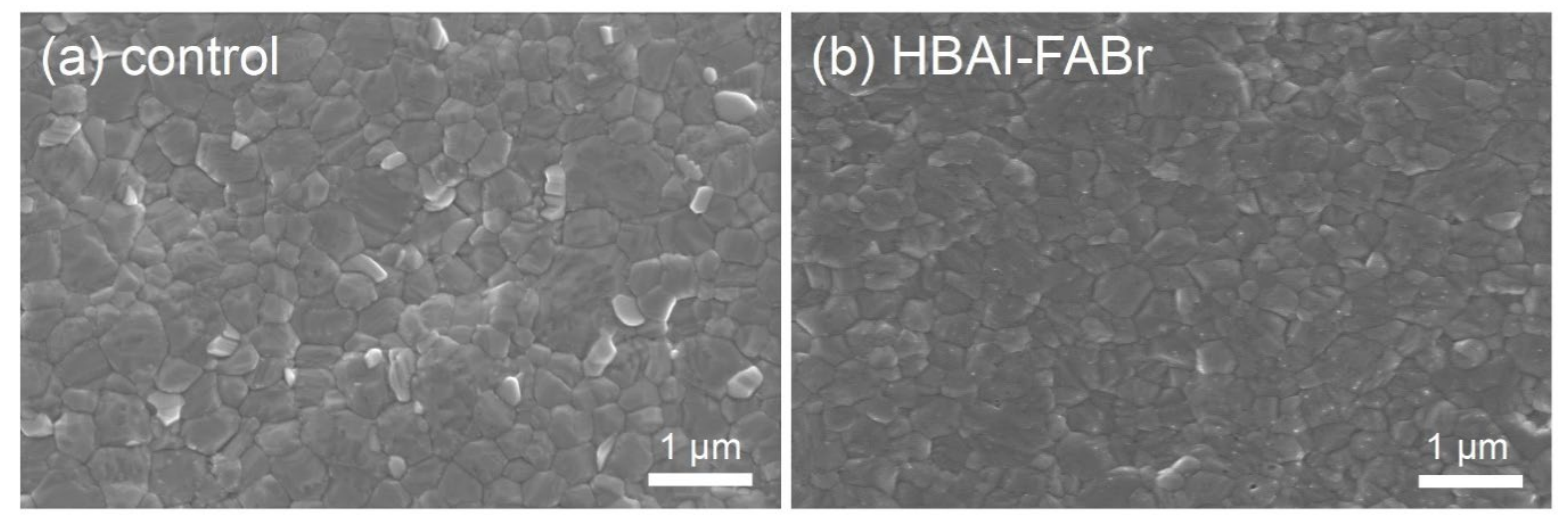

Figure 4 Surface HR-SEM images of the perovskite film (a) without and (b) with the posttreatment by $\mathrm{HBAI} \cdot \mathrm{FABr}$ for the passivation layer.

The extinct $\mathrm{PbI}_{2}$ by $\mathrm{HBAI} \cdot \mathrm{FABr}$ was also evidenced by X-ray diffraction (XRD) patterns where the $\mathrm{PbI}_{2}$ peak shown in the control film at $12.6^{\circ}$ readily disappears by the post-treatment with $\mathrm{HBAI} \cdot \mathrm{FABr}$, implying the reaction between the remaining $\mathrm{PbI}_{2}$ and the mixed-salt (Figure 5). Notably, the surface reaction with $\mathrm{PbI}_{2}$ is dominantly derived by $\mathrm{FABr}$ rather than HBAI. The exclusive use of HBAI for the post-treatment remains the $\mathrm{PbI}_{2}$ peak, indicating a difficulty of direct reaction between $\mathrm{PbI}_{2}$ and $\mathrm{HBAI}$ as shown in Figure S3. Cross-sectional SEM with elemental mapping by energy dispersive X-ray (EDX) was measured to examine the depthdependent element distribution (Figure S4). The EDX mapping demonstrates that fluorine, introduced by HBAI, is mainly located on the top of the 3D perovskite film, underlying that HBAI FABr forms a thin passivation layer on the surface. It is interesting to note that the distribution of $\mathrm{Br}$ is more biased toward the upper film, which is attributed to the contribution from the post-treated HBAI·FABr as well as the tendency of $\mathrm{Br}$ being prone to accumulate near the upper layer in the mixed-halide perovskite film. ${ }^{33}$

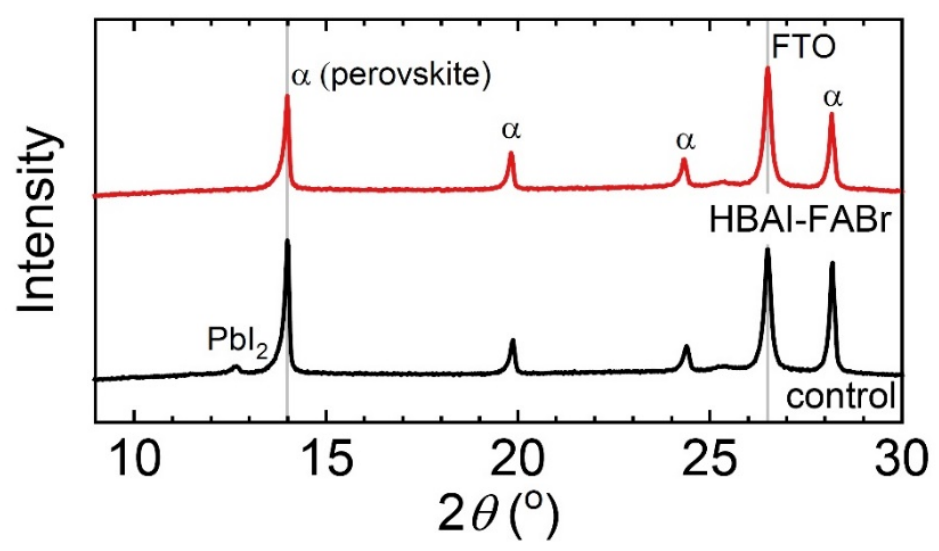


Figure 5 XRD patterns of control perovskite film (black) and the post-treated film with $\mathrm{HBAI} \cdot \mathrm{FABr}$ for the passivation layer (red).

In XRD results, it is suggested that the reaction with excess PbI2 mainly undergoes by FABr. Therefore, ${ }^{1} \mathrm{H}$-nuclear magnetic resonance $\left({ }^{1} \mathrm{H}-\mathrm{NMR}\right)$ measurements of HBAI, FABr and HBAI FABr mixed-salt were conducted to further understand the role of HBAI in the mixedsalts system from the perspective of the molecular interactions (Figure 6(a), full spectra in Figure S5). Notably, HBAI containing seven F atoms is easy to form hydrogen-bonding with active protons in the amino group in $\mathrm{FABr}$ due to the strong electronegativity. In order to classify different proton signals of the mixed-salt, ${ }^{1} \mathrm{H}-{ }^{1} \mathrm{H}$ COSY spectroscopy was demonstrated, which is an efficient method to determine spin-spin coupling correlations between neighboring protons, as shown in Figure 6(b). According to the cross peaks in the spectra, the signal peak $(8.90 \mathrm{ppm})$ corresponding to the amino groups of $\mathrm{FABr}$ splits into three peaks (9.02 ppm, $8.72 \mathrm{ppm}$, and $8.69 \mathrm{ppm}$, respectively) in the mixed-salt of $\mathrm{HBAI} \cdot \mathrm{FABr}$. Furthermore, a downfield shift of the $\mathrm{NH}_{3}{ }^{+}$peak is observed from $8.74 \mathrm{ppm}$ in HBAI to 8.83 ppm in HBAI·FABr. These obvious chemical shifts are attributed to a change in the hydrogen nucleus electron cloud density caused by atomic interaction. The strong H-F hydrogen-bonding can stabilize the movable $\mathrm{FA}^{+}$cation to form a stable interlayer on top of the $3 \mathrm{D}$ perovskite, which would effectively help to reduce the surface defects and suppress the carrier recombination, ${ }^{34}$ as discussed later.

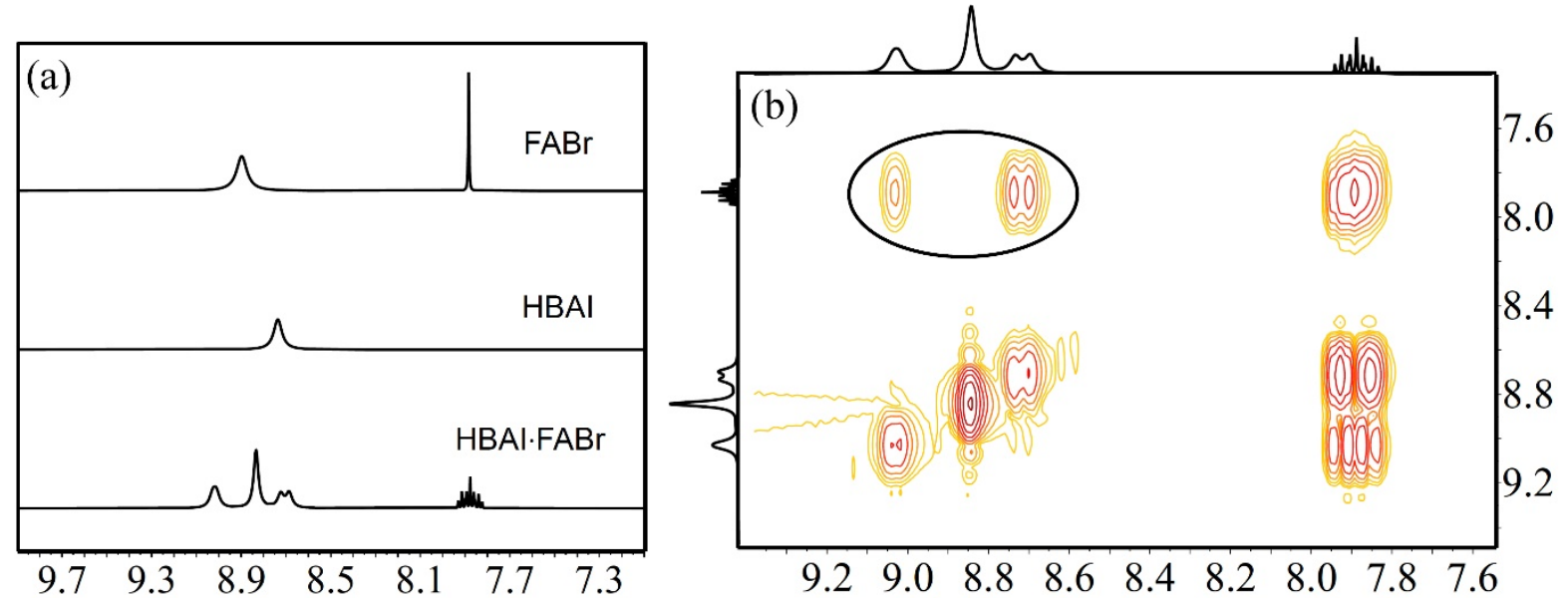

Figure 6 (a) ${ }^{1} \mathrm{H}-\mathrm{NMR}$ spectra of FABr, HBAI and mixed salts of HBAI $\mathrm{FABr}$ (molar ratio = $1: 1$ ) in DMSO; (b) ${ }^{1} \mathrm{H}-{ }^{1} \mathrm{H}$ COSY spectrum of mixed salts of $\mathrm{HBAI} \cdot \mathrm{FABr}$ (molar ratio $=1: 1$ ) in 
DMSO.

To analyze the passivation role of $\mathrm{HBAI} \cdot \mathrm{FABr}$, we carried Density Functional Theory (DFT) simulations of a 3D $\mathrm{MAPbI}_{3}$ perovskite slab, which fully covered by HBAI FABr. By applying a procedure previously reported (see Supporting Information for Computational Details), ${ }^{35}$ a $\mathrm{PbI}_{2}$-rich perovskite surface was set as a control and added one layer of $\mathrm{FABr}$, where the $\mathrm{Br}$ species are bonded to the undercoordinated $\mathrm{Pb}$ atoms. We subsequently considered the interaction of an HBAI layer on top of the perovskite-bound FABr layer. The HBAI FABr interaction is found to take place in this case through the HBAI fluorine atoms and the hydrogen atoms of FABr, as shown in Figure 7.

a)

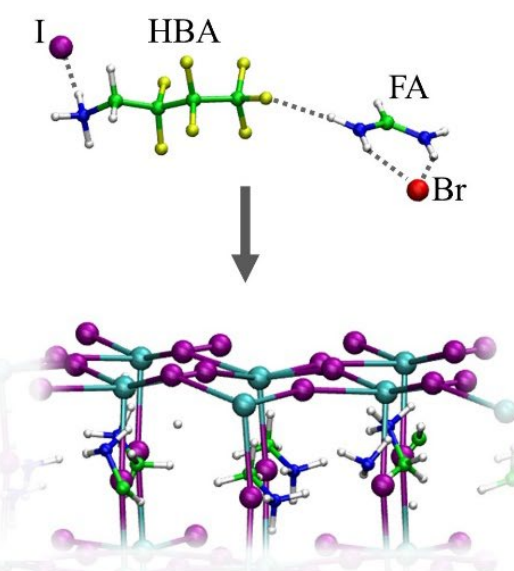

b)

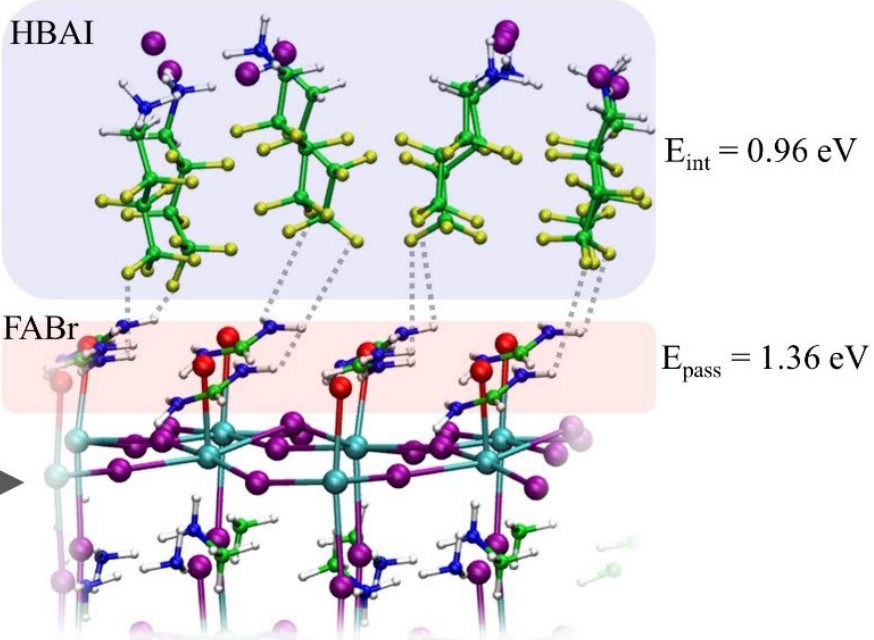

Figure 7. (a) HBAI-FABr interacting molecules (above) and the $\mathrm{PbI}_{2}$-rich perovskite slab; (b) HBAI-FABr-passivated perovskite interface.

To evaluate the stability of the covering layer, we calculated the passivation energy of $\mathrm{FABr}$

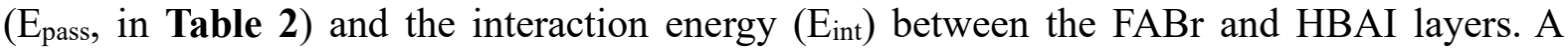
passivation process with $\mathrm{E}_{\mathrm{pass}}=1.36 \mathrm{eV} /$ molec and a remarkable value of $\mathrm{E}_{\text {int }}(0.96 \mathrm{eV})$ were calculated, underlying a favorable interaction between FABr and HBAI, in line with the ${ }^{1} \mathrm{H}$ NMR measurements. These values increase to 1.83 and $1.32 \mathrm{eV}$ when including dispersion interactions through Grimme's D3 approach. ${ }^{36}$ These results confirm the possibility of the $\mathrm{HBAI} \cdot \mathrm{FABr}$ interaction at the perovskite interface and confirm the spontaneous tendency to 
form a passivating film. To fully characterize the energetics of the passivation process, we also calculated the $\mathrm{E}_{\text {pass }}$ of HBAI, HBABr, MAI and FAI, (Table 2). FAI shows a similar $\mathrm{E}_{\text {pass }}$ with respect to $\mathrm{FABr}$, whereas $\mathrm{HBAI}$ and $\mathrm{HBABr}$ have a higher passivation energy, indicating that also these salts are expected to interact with the undercoordinated $\mathrm{Pb}$ atoms of the surface and to play a role in the passivation process of the surface traps. Moreover, this important interaction provided by HBAI can be associated to a better device stability. Furthermore, the effect of passivating layers on the electronic properties of the composite systems was analyzed. As shown in the density of state reported in Figure S6, by adding the passivation layer, the trap states associated to the under coordinated $\mathrm{Pb}$ on the surface are completely removed, in line with the increasing of $V_{\mathrm{OC}}$. As previously reported, ${ }^{35}$ the percentage of passivating molecular coverage can drastically affect the ionization potential and up-shifts the VB to a more positive value. Moreover, the MAI excess up-shifts the VB of the perovskite, leading to an unfavorable mismatch of the energy level with respect to HTM with regards to the hole extraction process. To evaluate the role of $\mathrm{HBAI} \cdot \mathrm{FABr}$ treatment in determining the VB perovskite shift, we thus calculated the ionization potential of the passivated perovskite, shown in Table 2 . The ionization potential of the HBAI-FABr-terminated system shows a slightly more positive ionization potential $(4.9 \mathrm{eV})$ with respect to that obtained for complete MAI passivation (4.7 $\mathrm{eV})$. Moreover, the direct passivation of HBAI leads to a higher ionization potential $(5.2 \mathrm{eV})$. This result is very interesting because of the full passivation with HBAI FABr layer and with HBAI, which leads to a slight VB down-shift with respect to MAI passivation, ensures at the same time an efficient hole extraction process ${ }^{35}$ and a reduction of surface traps.

Table 2 Ionization potentials (eV) for the investigated species and passivation energy.

\begin{tabular}{cccc} 
Termination & $\begin{array}{c}\text { Ionization } \\
\text { potential }(\mathrm{IE}) \\
(\mathrm{eV})\end{array}$ & $\begin{array}{c}\text { Passivation energy } \\
\text { per molecule } \\
(\mathrm{eV})\end{array}$ & $\begin{array}{c}\text { Passivation energy per } \\
\text { molecule (eV) - D3 } \\
\text { Interaction }\end{array}$ \\
\hline MAI & 4.7 & 1.64 & \\
FAI & 4.8 & 1.36 & 1.83 \\
FABr & 4.9 & 1.36 & 2.19 \\
HBAI & 5.2 & 1.61 & 1.32 \\
HBABr & 5.3 & 1.57 & \\
HBAI on FABr* & 4.9 & 0.96 & \\
PbI $_{2}$ & 6.4 & - &
\end{tabular}

*This is not a passivation energy but the interaction energy ( $\left.\mathrm{E}_{\mathrm{int}}\right)$ between FABr-terminated slab and one HBAI monolayer through $\mathrm{H} \cdots \mathrm{F}$ hydrogen bond. 
To confirm the proposed passivation effect by the mixed-salts system, further characterization on charge recombination and related trap density was carried out. The control and the posttreated perovskite films were prepared on glass substrates for photoluminescence (PL) measurements to investigate the effect of HBAI·FABr-based passivation layer on the charge recombination behavior. In Figure 8(a), TR-PL spectra are shown and fitted with a second order exponential decay curve where the longer time constant is responsible for radiative charge recombination. ${ }^{37}$ The time constant of the $3 \mathrm{D}$ perovskite film is increased by $25 \%$ from $1.33 \mu \mathrm{s}$ to $1.66 \mu \mathrm{s}$ due to the HBAI·FABr-based passivation layer, coming along with increased PL signal in the films. In Figure 8(b), steady-state PL and electroluminescence (EL) spectra are shown, which have been measured on complete devices. Whereas the spectra do not change with passivation, the luminescence intensities increase. Remarkably, the EL intensity (left axis) increases by a factor of 10 , which is consistent with the increase of $V_{\mathrm{OC}}$ (for the devices under investigation here ca. $60 \mathrm{mV}),{ }^{38}$ whereas the PL is only slightly enhanced (right axis). This is a strong evidence that the post-treatment with $\mathrm{HBAI} \cdot \mathrm{FABr}$ mainly acts on reducing the surface recombination. ${ }^{39}$ The overall improved luminescence by the post-treatment with HBAI FABr indicates the pronounced radiative recombination by suppressing the trap-assisted nonradiative recombination in the presence of the passivation layer. ${ }^{10,40}$ The dark I-V was measured to calculate the trap-filled limit voltage $\left(V_{\mathrm{TFL}}\right)$ and thus estimate the trap density $(\mathrm{Nt})$ to assure the passivation effect by the mixed salts of HBAI·FABr (Figure S7). A control device shows $0.67 \mathrm{~V}$ of $V_{\mathrm{TFL}}$, which is decreased to $0.53 \mathrm{~V}$ by employing the passivation layer. $N_{\mathrm{t}}$ is estimated by the following equation, ${ }^{41}$

$$
V_{T F L}=\frac{\mathrm{e} N_{t} d^{2}}{2 \varepsilon_{0} \varepsilon}
$$

where $\mathrm{e}$ is the elementary charge, $\mathrm{d}$ is the perovskite film thickness, $\varepsilon_{0}$ is the vacuum permittivity, and $\varepsilon$ is the relative dielectric constant. It is found that the surface passivation by HBAI FABr leads to a decrease in $N_{\mathrm{t}}$ from $1.84 \cdot 10^{16} \mathrm{~cm}^{-3}$ to $1.46 \cdot 10^{16} \mathrm{~cm}^{-3}$, which is in the reasonable range for perovskite. ${ }^{42-44}$ This suggests the formation of an effective passivation layer by the preferential reaction between $\mathrm{FABr}$ and the excessive $\mathrm{PbI}_{2}$, respectively from the HBAI FABr mixed-salts and the 3D perovskite, which is followed by the internal interaction as confirmed by ${ }^{1} \mathrm{H}-\mathrm{NMR}$. 

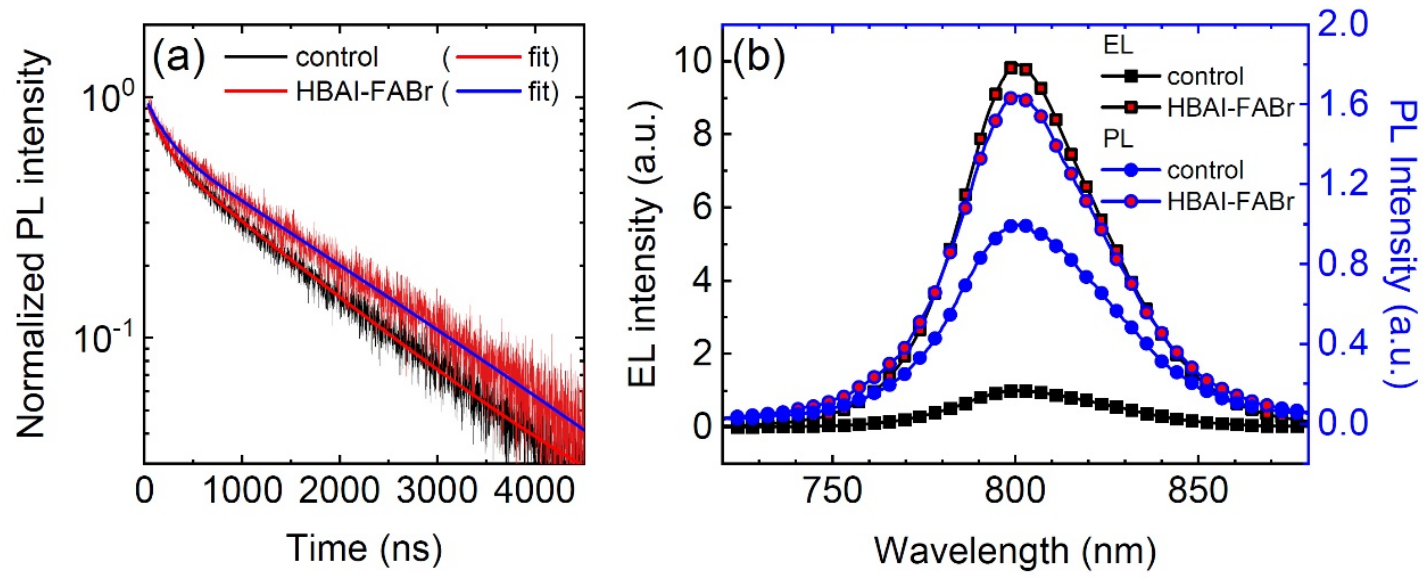

Figure 8 (a) TR-PL of perovskite film with (red) and without (black) a passivation layer of HBAI FABr. Thick blue and red lines indicate the fitted results with and without the passivation layer, respectively. (b) Luminescence spectra of complete devices. The intensity is given referred to the peak maximum of the control device for EL (left axis, black lines) and PL (right axis, blue lines).

The stability of the devices was monitored in ambient air without any encapsulation for $500 \mathrm{~h}$ to see the effect of passivation layer on long-term stability. As shown in Figure 9, control device exhibits overall good long-term stability, showing a 10\% loss in PCE mostly due to the apparent reduction in $\mathrm{FF}$, while $J_{\mathrm{SC}}$ and $V_{\mathrm{OC}}$ are comparably stable for $500 \mathrm{~h}$. Remarkably, the device with the HBAI·FABr-based passivation layer results in an outstanding stability even showing a $2 \%$ increase in PCE after $500 \mathrm{~h}$ due to the slightly enhanced FF, which is completely opposite to the control device without the passivation layer. The stable FF observed from the $\mathrm{HBAI} \cdot \mathrm{FABr}$ device is likely to originate from the strong hydrogen-bonding of the mixed salts composing the passivation layer. Similarly, the HBAI·FABr device demonstrated higher operational stability compared to the control device at mpp under full sun intensity in $\mathrm{N}_{2}$ condition. While PCE of the control device dropped by $10 \%$ for $450 \mathrm{~h}$, the HBAI FABr-based device retains $95 \%$ of the initial PCE (Figure S8). Furthermore, the enhanced moisture resistance by multiple $\mathrm{F}$ atoms on HBAI is evidenced by increased contact angle of water droplet on the perovskite surface from $49.8^{\circ}$ for control film to $66.8^{\circ}$ for HBAI·FABr-treated film (Figure S9). 


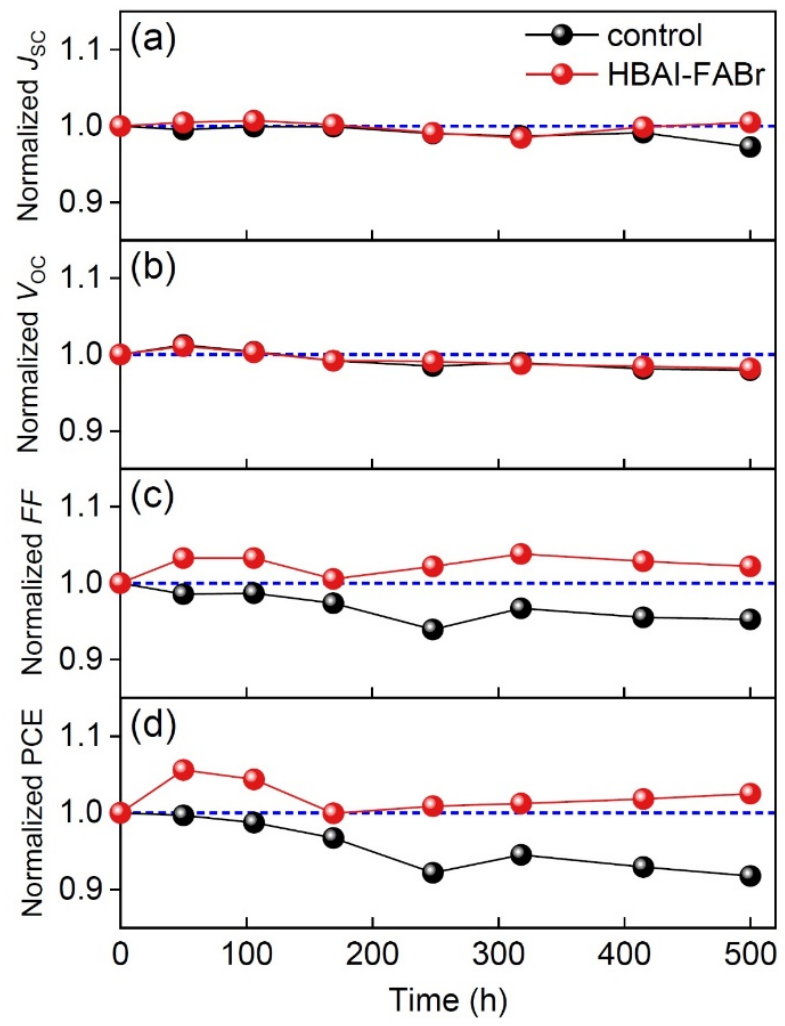

Figure 9 Stability of the optimized device (red) with the mixed passivation layer of HBAI FABr compared to the control device (black) without the passivation layer. The device storage condition was R.H. $<20 \%$ in dark at room temperature.

In conclusion, a new ammonium salt, HBAI, is adopted coupled with FABr in a mixedsalt form to compose a passivation layer on top of the 3D perovskite bulk film. The use of mixed-HBAI-FABr facilitates the internal interactions between HBAI and FABr through hydrogen bonding, which plays a key role in building the optimized interlayer in terms of defect passivation, energy level alignment, and stability. Devices employing the passivation layer results in outstanding photovoltaic properties, particularly in open-circuit voltage and fill factor compared to control devices without the passivation layer. On one hand, the effect of $\mathrm{HBAI} \cdot \mathrm{FABr}$ is reflected in the suppressed charge recombination by passivating the surface traps of the 3D perovskite bulk film, as evidenced by the increased PL and EL intensity. Calculated passivation energy by employing the mixed-HBAI·FABr salt is well in accordance with the observed results implying the effectively suppressed non-radiative recombination. On the other hand, the efficient hole extraction is enabled by well-matched energy level with respect to HTM. The employment of the passivation layer is indeed helpful for the long-term 
stability by providing more stable interface with reduced defects, leading to an ensured $500 \mathrm{~h}$ stability.

\section{Experimental}

\section{Synthesis of HBAI}

2,2,3,3,4,4,4-heptafluorobutan-1-amine (1g, $5.0 \mathrm{mmol}$ ) (across, 97\%) was dissolved in $30 \mathrm{~mL}$ $\mathrm{MeOH}$ at $0{ }^{\circ} \mathrm{C}$, hydroiodic acid solution $(1 \mathrm{~mL}$ ) (across, $57 \%$ in water) was added dropwise and the solution was stirred for $1 \mathrm{~h}$, then the mixture was removed to room temperature and stirred for another $2 \mathrm{~h}$. After solvent evaporation, the resulted solid residue was washed with diethyl ether three times, affording white solid product( $1.40 \mathrm{~g}, 86 \%$ yield $).{ }^{1} \mathrm{H}$ NMR $(400 \mathrm{MHz}$, DMSO) $\delta 8.74(\mathrm{~s}, 3 \mathrm{H}), 4.02(\mathrm{t}, \mathrm{J}=17.7 \mathrm{~Hz}, 2 \mathrm{H})$.

\section{Substrate}

Fluorine doped tin oxide (FTO) substrates (NSG-10) were chemically etched by zinc powder and $4 \mathrm{M} \mathrm{HCl}$ solution and sonicated in $2 \%$ Hellmanex water solution for $30 \mathrm{~min}$, acetone for $15 \mathrm{~min}$ and ethanol for $15 \mathrm{~min}$, respectively. Then, all substrates were further cleaned by UVOzone for $15 \mathrm{~min}$. Then, a compact $\mathrm{TiO}_{2}$ layer was deposited on cleaned FTO substrates via spray pyrolysis deposition from a precursor solution of titanium diisopropoxide bis(acetylacetonate) in anhydrous ethanol, with oxygen as carrier gas. Substrates were heated at $450{ }^{\circ} \mathrm{C}$ and kept at this temperature for $15 \mathrm{~min}$ before and $30 \mathrm{~min}$ after the spray of the precursor solution, then left to cool down to room temperature. Mesoporous $\mathrm{TiO}_{2}$ layer was spin-coated at $4000 \mathrm{rpm}$ for $20 \mathrm{~s}$, with the acceleration rate of $2000 \mathrm{rpm} / \mathrm{s}$, using a $30 \mathrm{~nm} \mathrm{TiO}_{2}$ paste (Dyesol 30 NR-D) diluted in ethanol with 1:6 volume ratio. After the spin-coating, the substrates were dried at $80{ }^{\circ} \mathrm{C}$ for $10 \mathrm{~min}$ and then sintered at $450{ }^{\circ} \mathrm{C}$ for 30 min under dry air flow.

\section{Perovskite layer}

The perovskite precursor solution was prepared by dissolving a mixture of cesium iodide $(0.07$ mmol, TCI Co. Ltd.), methylammonium bromide (0.14 mmol, Dyenamo), formamidinium iodide (1.19 mmol, Dyenamo), lead iodide (1.45 mmol, Alfa Co. Ltd.) in $1 \mathrm{~mL}$ mixure of DMF and DMSO (DMF:DMSO=4:1 v/v, Acros). The perovskite solution was spin-coated through two-step program (1000 rpm for $10 \mathrm{~s}$ and $6000 \mathrm{rpm}$ for $20 \mathrm{~s}$ ) with pouring chlorobenzene as an anti-solvent $5 \mathrm{~s}$ before the end of the second step. Then the substrates were annealed at $100{ }^{\circ} \mathrm{C}$ for $1 \mathrm{~h}$ in dry air. The mixed passivation layer was spin-coated at $4000 \mathrm{rpm}$ for $20 \mathrm{~s}$ on the asprepared perovskite films and dried on a hot plate at $80{ }^{\circ} \mathrm{C}$ for $10 \mathrm{~min}$. 


\section{Hole transporting layer and Au top contact}

The substrates were cooled down to room temperature after annealing the perovskite. A spirofluorene-linked methoxy triphenylamines (spiro-MeOTAD, Merck) solution was deposited by spin coating at $4000 \mathrm{rpm}$ for $20 \mathrm{~s}$, as hole-transporting material. $90 \mathrm{mg}$ spiroMeOTAD was dissolved in $1 \mathrm{ml}$ chlorobenzene, doped by $20.625 \mu \mathrm{L}$ bis(trifluoromethylsulfonyl)imide lithium salt solution $(520 \mathrm{mg} / \mathrm{mL}$ LiTFSI in acetonitrile), and $35.5 \mu \mathrm{L}$ 4-tert-butylpyridine (tBP, Sigma-Aldrich). Finally, $80 \mathrm{~nm}$ of Au top electrode was deposited through thermal evaporator under high vacuum with an active area of $0.16 \mathrm{~cm}^{2}$.

\section{Characterization}

The solar cell devices were measured using a $300 \mathrm{~W}$ Xenon light source (Oriel). The spectral mismatch between AM 1.5 G and the solar simulator was calibrated by a Schott K113 Tempax filter (Prazosopms G;as \& Optik $\mathrm{GmbH}$ ). The light intensity was calibrated with a silicon photodiode with an IR-cutoff filter (KG2, Schott). Current-voltage characteristics were applied by an external voltage bias while measuring the corresponding current with Keithley 2400 . The voltage scan rate was $50 \mathrm{mV} / \mathrm{s}$. The devices were covered with a black metal mask with an active area of $0.16 \mathrm{~cm}^{2}$. Incident photon to current efficiency (IPCE) was carried by a commercial apparatus (Aekeo-Ariadne, Cicci Research s.r.l.). The top-view and cross-section morphologies of the samples was characterized using a high-resolution scanning electron microscope (Zeiss Merlin) with an in-lens secondary electron detector. The X-ray diffraction patterns were recorded with PANalytical Empyrean system with a PIXcel-1D detector, BraggBrentano beam optics and parallel beam optics. Light source is from copper $\mathrm{K} \alpha$ beam filtered with nickel $\beta$ filter. Diffraction spectra were characterized between 2-theta of $10^{\circ}$ and $70^{\circ}$ at a scan rate of $1^{\circ}$ per minute with the step width of $0.02^{\circ}$. UV-Vis measurements were performed on a Varian Cary 500 spectrometer (Varian USA). Steady-state photoluminescence (PL) and electroluminescence (EL) spectra were obtained with an Andor Kymera 193i spectrometer equipped with a silicon CCD camera (iDus DU240A-OE). Excitation was provided by a CW laser (Obis, $422 \mathrm{~nm}, 1 \mathrm{~mW}$, beam diameter at 1/e2 $0.9 \pm 0.1 \mathrm{~mm}$ ) for PL. For EL, a current of $0.5 \mathrm{~mA}$ (ca. $2 \mathrm{~mA} / \mathrm{cm} 2$ ) was applied with a Biologic SP-300 potentiostat. Time-resolved photoluminescence (TRPL) was measured by using a picosecond pluse diode laser (EPL-405) with an excitation wavelength of $405 \mathrm{~nm}$, pulse width of $49 \mathrm{ps}$. ${ }^{1} \mathrm{H}$ NMR measurements were performed on Bruker AvanceIII-400 MHz NMR spectrometer. Operational stability meas urements; SCLC measurements 


\section{Conflicts of interest}

There are no conflicts to declare.

\section{Supporting Information}

The Supporting Information is available free of charge on the ACS Publications website at DOI: XXXX

Statistical box charts depending on the ratio between HBAI and FABr in the mixed-salt; IPCE spectra of the control devices and HBAI·FAI, HBAI·FABr, and HBAI·FACl-treated devices; XRD patterns of the post-treated film with HBAI; cross-sectional SEM image and corresponding EDX mapping; ${ }^{1} \mathrm{H}-\mathrm{NMR}$ raw spectra; Isodensity contour plots of DOS layer by layer; I-V response of the electron-only devices employing control perovskite film and the posttreated film with HBAI·FABr; Operational stability; contact angles; computational details.

\section{Acknowledgements}

We acknowledge N. Lucie for helping the SEM-EDX analysis, P. Mettraux for the XPS measurement. This project has received funding from the European Union's Horizon 2020 research and innovation programme under grant agreement No 764047 and from Swiss National Science Foundation for financial support with Project No. 200020_185041. This work was supported by the National Research Foundation of Korea(NRF) grant funded by the Korea government(MSIT) (No. 2020R1F1A1075558).

\section{References}

(1) H.-S. Kim, C.-R. Lee, J.-H. Im, K.-B. Lee, T. Moehl, A. Marchioro, S.-J. Moon, R. Humphry-Baker, J.-H. Yum, J. E. Moser, M. Grätzel and N.-G. Park, Lead Iodide Perovskite Sensitized All-Solid-State Submicron Thin Film Mesoscopic Solar Cell with Efficiency Exceeding 9\%. Sci. Rep., 2012, 2, 591.

(2) M. M. Lee, J. Teuscher, T. Miyasaka, T. N. Murakami and H. J. Sanith, Efficient Hybrid Solar Cells Based on Meso-Superstructured Organometal Halide Perovskites. Science., 2012, 338, 643-647.

(3) A. Kojima, K. Teshima, Y. Shirai and T. Miyasaka, Organometal Halide Perovskite as Visible-Light Sensitizers for Photovoltaic Cells. J. Am. Chem. Soc., 2009, 131, 6050-6051.

(4) N.-G. Park, Perovskite Solar Cells: An Emerging Photovoltaic Technology. Mat. Today., 2015, 18, 65-72. 
(5) K. Wang, D. Yang, C. Wu, M. Sanghadasa and S. Priya, Recent Progress in Fundamental Understanding of Halide Perovskite Semiconductors. Prog. Mater. Sci., 2019, 106, 100580.

(6) H. S. Kim, A. Hagfeldt and N.-G. Park, Morphological and Compositional Progress in Halide Perovskite Solar Cells. Chem. Commun., 2019, 55, 1192-1200.

(7) W. Du, S. Zhang, Q. Zhang and X. Liu, Recent Progress of Strong Exciton-Photon Coupling in Lead Halide Perovskites. Adv. Mater., 2019, 31, 1804894.

(8) L. Chouhan, S. Ghimire, C. Subrahmanyam, T. Miyasaka and V. Biju, Synthesis, Optoelectronic Properties and Applications of Halide Perovskites. Chem. Soc. Rev., 2020, 49, 1869-2885.

(9) Best Research-Cell Efficiencies Chart (https:/www.nrel.gov/pv/assets/pdfs/best-researchcell-efficiencies.20200406.pdf). Accessed on May 17, 2020.

(10) D. Luo, R. Su, W. Zhang, Q. Gong and R. Zhu, Minimizing Non-Radiative Recombination Losses in Perovskite Solar Cells. Nat. Rev. Mater., 2020, 5, 44-60.

(11) C. M. Wolff, P. Caprioglio, M. Stolterfoht and D. Neher, Nonradiative Recombination in Perovskite Solar Cells: The Role of Interfaces. Adv. Mater., 2019, 31, 1902762.

(12) W. Tress, Perovskite Solar Cells on the Way to Their Radiative Efficiency Limit - Insights into a Success Story of High Open-Circuit Voltage and Low Recombination. Adv. Energy Mater., 2017, 7, 1602358.

(13) T.-H. Han, S. Tan, J. Xue, L. Meng, J.-W. Lee and Y. Yang, Interface and Defect Engineering for Metal Halide Perovskite Optoelectronic Devices. Adv. Mater., 2019, 31, 1803515 .

(14) C. Ran, J. Xu, W. Gao, C. Huang and S. Dou, Defects in Metal Triiodide Perovskite Materials Towards High-Performance Solar Cells: Origin, Impact, Characterization, and Engineering. Chem. Soc. Rev., 2018, 47, 4581-4610.

(15) S. Akin, N. Arora, S. M. Zakeeruddin, M. Grätzel, R. H. Friend and M. I. Dar, New Strategies for Defect Passivation in High-Efficiency Perovskite Solar Cells. Adv. Energy Mater., 2020, 10, 1903090.

(16) E. Aydin, M. De Bastiani and S. De Wolf, Defect and Contact Passivation for Perovskite Solar Cells. Adv. Mater., 2019, 31, 1900428.

(11) M. Saliba, T. Matsui, J-Y. Seo, K. Domanski, J-P. Correa-Baena, M. K. Nazeeruddin, S. M. Zakeeruddin, W. Tress, A. Abate, A. Hagfeldt and M. Grätzel, Cesium-containing Triple Cation Perovskite Solar Cells: Improved Stability, Reproducibility and High Efficiency. Energy Environ. Sci., 2016, 9, 1989-1997. 
(18) D.-Y. Son, S.-G. Kim, J.-Y. Seo, S.-H. Lee, H. Shin, D. Lee and Park, N.-G. Universal Approach toward Hysteresis-Free Perovskite Solar Cell via Defect Engineering. J. Am. Chem. Soc., 2018, 140, 1358-1364.

(19) A. Krishna, S. Gottis, M. K. Nazeeruddin and F. Sauvage, Mixed Dimensional 2D/3D Hybrid Perovskite Absorbers: The Future of Perovskite Solar Cells? Adv. Funct. Mater., 2019, 29, 1806482.

(20) R. Wang, J. Xue, K-L. Wang, Z-K. Wang, Y. Luo, D. Fenning, G. Xu, S. Nuryyeva, T. Huang, Y. Zhao, J.Y. Yang, J. Zhu, M. Wang, S. Tan, I. Yavuz, K. N. Houk and Y. Yang, Constructive Molecular Configurations for Surface-defect Passivation of Perovskite Photovoltaics. Science., 2019, 366, 1509-1513.

(21) S. Yang, S. Chen, E. Mosconi, Y. Fang, X. Xiao, C. Wang, Y. Zhou, Z. Yu, J. Zhao, Y. Gao, F. De Angelis and J. Huang, Stabilizing Halide Perovskite Surfaces for Solar Cell Operation with Wide-bandgap Lead Oxysalts. Science., 2019, 365, 473-478.

(22) D. H. Cao, C. C. Stoumpos, C. D. Malliakas, M. J. Katz, O. K. Farha, J. T. Hupp and M. G. Kanatzidis, Remnant $\mathrm{PbI}_{2}$, an Unforeseen Necessity in High-Efficiency Hybrid PerovskiteBased Solar Cells? APL Mater., 2014, 2, 091101.

(23) S. Pathak, A. Sepe, A. Sadhanala, F. Deschler, A. Haghighirad, N. Sakai, K. C. Goedel, S. D. Stranks, N. Noel, M. Price, S. Hüttner, N. A. Hawkins, R. H. Friend, U. Steiner and H. J. Snaith, Atmospheric Influence upon Crystallization and Electronic Disorder and Its Impact on the Photophysical Properties of Organic-Inorganic Perovskite Solar Cells. ACS Nano., 2015, 9, 2311-2320.

(24) D. Bi, W. Tress, M. I. Dar, P. Gao, J. Luo, C. Renevier, K. Schenk, A. Abate, F. Giordano, J-P. Correa Baena, J-D. Decoppet, S. M. Zakeeruddin, M. K. Nazeeruddin, M. Grätzel and A. Hagfeldt, Efficient Luminescent Solar Cells Based on Tailored Mixed-cation Perovskites. Sci. $A d v ., 2016,2$, e1501170.

(25) Q. Chen, H. Zhou, T-B. Song, S. Luo, Z. Hong, H-S. Duan, L. Dou, Y. Liu and Y. Yang, Controllable Self-Induced Passivation of Hybrid Lead Iodide Perovskites toward High Performance Solar Cells. Nano Lett., 2014, 14, 4158-4163.

(26) Q. Jiang, Y. Zhao, X. Zhang, X. Yang, Y. Chen, Z. Chu, Q. Ye, X. Li, Z. Yin and J. You, Surface Passivation of Perovskite Films for Efficient Solar Cells. Nat. Photonics., 2019, 13, 460-466.

(27) Y. Liu, S. Akin, L. Pan, R. Uchida, N. Arora, J. Milić, A. Hinderhofer, F. Schreiber, A. R. Uhl, S. M. Zakeeruddin, A. Hagfeldt, M. I. Dar and M. Grätzel, Ultrahydrophobic 3D/2D 
Fluoroarene Bilayer-Based Water-Resistant Perovskite Solar Cells with Efficiencies Exceeding 22\%. Sci. Adv., 2019, 5, eaaw2543.

(28) H. Kim, S.-U. Lee, D. Y. Lee, M. J. Paik, H. Na, J. Lee and S. I. Seok, Optimal Interfacial Engineering with Different Length of Alkylammonium Halide for Efficient and Stable Perovskite Solar Cells. Adv. Energy Mater., 2019, 9, 1902740.

(29) J. J. Yoo, S. Wieghold, M. C. Sponseller, M. R. Chua, S. N. Bertram, N. T. P. Hartono, J. S. Tresback, E. C. Hansen, J-P. Correa-Baena, V. Bulović, T. Buonassisi, S. S. Shin and M. D. Bawendi, An Interface Stabilized Perovskite Solar Cell with High Stabilized Efficiency and Low Voltage Loss. Energy Environ. Sci., 2019, 12, 2192-2199.

(30) H. Zhu, Y. Liu, F.T. Eickemeyer, L. Pan, D. Ren, M. A. Ruiz-Preciado, B. Carlsen, B. Yang, X. Dong, Z. Wang, H. Liu, S. Wang, S. M. Zakeeruddin, A. Hagfeldt, M. I. Dar, X. Li and M. Grätzel, Tailored Amphiphilic Molecular Mitigators for Stable Perovskite Solar Cells with 23.5\% Efficiency. Adv. Mater., 2020, 32, 1907757.

(31) Y. Cho, A. M. Soufiani, J. S. Yun, J. Kim, D. S. Lee, J. Seidel, X. Deng, M. A. Green, S. Huang and A. W. Y. Ho-Baillie, Mixed 3D-2D Passivation Treatment for Mixed-Cation Lead Mixed-Halide Perovskite Solar Cells for Higher Efficiency and Better Stability. Adv. Energy Mater., 2018, 8, 1703392.

(32) M. M. Tavakoli, P. Yadav, D. Prochowicz, M. Sponseller, A. Osherov, V. Bulović and J. Kong, Controllable Perovskite Crystallization via Antisolvent Technique Using Chloride Additives for Highly Efficient Planar Perovskite Solar Cells. Adv. Energy Mater., 2019, 9, 1803587.

(33) K. T. Cho, S. Paek, G. Grancini, C. Roldán-Carmona, P. Gao, Y. Lee and M. K. Nazeeruddin, Highly Efficient Perovskite Solar Cells with a Compositionally Engineered perovskite/Hole transporting Material Interface. Energy Environ. Sci., 2017, 10, 621-627.

(34) C.-C. Zhang, Z.-K. Wang, S. Yuan, R. Wang, M. Li, M. F. Jimoh, L.-S. Liao and Y. Yang, Polarized Ferroelectric Polymers for High-Performance Perovskite Solar Cells. Adv. Mater., 2019, 31, 1902222.

(35) D. Meggiolaro, E. Mosconi, A. H. Proppe, R. Quintero-Bermudez, S. O. Kelley, E. H. Sargent and F. De Angelis, Energy Level Tuning at the $\mathrm{MAPbI}_{3}$ Perovskite/Contact Interface Using Chemical Treatment. ACS Energy Lett., 2019, 4, 2181-2184.

(36) S. Grimme, J. Antony, S. Ehrlich and H. Krieg, A Consistent and Accurate ab initio Parametrization of Density Functional Dispersion Correction (DFT-D) for the 94 Elements HPu. J. Chem. Phys., 2010, 132, 154104. 
(37) P.-W. Liang, C.-Y. Liao, C.-C. Chueh, F. Zuo, S. T. Williams, X.-K. Xin, J. Lin and A. K.Y. Jen, Additive Enhanced Crystallization of Solution-Processed Perovskite for Highly Efficient Planar-Heterojunction Solar Cells. Adv. Mater., 2014, 26, 3748-3754.

(38) W. Tress, N. Marinova, O. Inganäs, M. K. Nazeeruddin, S. M. Zakeeruddin and M. Grätzel, Predicting the Open-Circuit Voltage of $\mathrm{CH}_{3} \mathrm{NH}_{3} \mathrm{PbI}_{3}$ Perovskite Solar Cells Using Electroluminescence and Photovoltaic Quantum Efficiency Spectra: the Role of Radiative and Non-Radiative Recombination. Adv. Energy Mater., 2015, 5, 1400812.

(39) P. Caprioglio, M. Stolterfoht, C. M. Wolff, T. Unold, B. Rech, S. Albrecht and D. Neher, On the Relation between the Open-Circuit Voltage and Quasi-Fermi Level Splitting in Efficient Perovskite Solar Cells. Adv. Energy Mater., 2019, 9, 1901631.

(40) W. Tress, M. Yavari, K. Domanski, P. Yadav, B. Niesen, J. P. Correa-Baena, A. Hagfeldt and M. Grätzel, Interpretation and Evolution of Open-Circuit Voltage, Recombination, Ideality Factor and Subgap Defect States during Reversible Light-Soaking and Irreversible Degradation of Perovskite Solar Cells. Energy Environ. Sci., 2018, 11, 151-165.

(41) Y. Liu, J. Sun, Z. Yang, D. Yang, X. Ren, H. Xu, Z. Yang and S. Liu. 20-mm-Large SingleCrystalline Formamidinium-Perovskite Wafer for Mass Production of Integrated Photodetectors. Adv. Optical Mater., 2016, 4, 1829-1837.

(42) N. Liu, Q. Du, G. Yin, P. Liu, L. Li, H. Xie, C. Zhu, Y. Li, H. Zhou, W.-B. Zhang and Q. Chen. Extremely low trap-state energy level perovskite solar cells passivated using $\mathrm{NH}_{2}$-POSS with improved efficiency and stability. J. Mater. Chem. A, 2018, 6, 6806-6814.

(43) M. Li, G. Cao and J. Tian. Monolithic $\mathrm{MAPbI}_{3}$ films for high-efficiency solar cells via coordination and a heat assisted process. J. Mater. Chem. A, 2017, 5, 21313-21319.

(44) H. Wang, Z. Wang, Z. Yang, Y. Xu, Y. Ding, L. Tan, C. Yi, Z. Zhang, K. Meng, G. Chen et al. Ligand-Modulated Excess $\mathrm{PbI}_{2}$ Nanosheets for Highly Efficient and Stable Perovskite Solar Cells. Adv. Mater., 2020, 32, 2000865. 